\section{Sperm-Specific microRNAs - Their Role and Function}

\author{
Maha Klahid AI-Gazi ${ }^{1,2}$ and Michael Carroll ${ }^{1,2 *}$ \\ ${ }^{1}$ School of Healthcare Science, Faculty of Science and Engineering, John \\ Dalton Building, Manchester Metropolitan University, Chester Street, \\ Manchester, M1 5GD, UK \\ ${ }^{2}$ Healthcare Science Research Centre, Faculty of Science and Engineer- \\ ing, John Dalton Building, Manchester Metropolitan University, Chester \\ Street, Manchester, M1 5GD, UK
}

\begin{abstract}
microRNAs (miRNAs) are small non-coding single-stranded molecules (containing about 22 nucleotides) that are widely found in many organisms, as varied as micro-organisms, plants, invertebrates and vertebrates. These miRNAs function in RNA silencing and post-transcriptional regulation of gene expression. miRNAs have been found to be involved in a number of biological and pathological processes, including sperm biology and infertility. This review will describe and discuss the role of miRNAs in terms of their role in sperm function, male infertility and gene regulation.
\end{abstract}

Keywords: Infertility; microRNAs; non-coding RNA; Sperm; Transgenerational inheritance

\section{Introduction}

The sperm cell is highly specialised, with the sole purpose to fertilize an oocyte. Sperm assumes a simplistic structure, consisting of a head, which is mostly void of cytoplasm, a nucleus packed with DNA tightly wound around protamines; a midpiece housing the mitochondria necessary to fuel its locomotion, and a tail or principle piece that whips in motion to move the sperm towards the oocyte. However, despite the lack of cytoplasmic space, increasing studies in sperm biology have revealed that the sperm cell contains a myriad of molecular constituents that may play a role post-fertilization, influencing the oocyte and embryo development (figure 1). One such group of molecules are the small non-coding RNAs, which include the microRNAs (miRNAs).

*Corresponding author: Michael Carroll, School of Healthcare Science, Faculty of Science and Engineering, John Dalton Building, Manchester Metropolitan University, Chester Street, Manchester, M1 5GD, UK, Tel: +44 1612471231; E-mail: michael.carroll@mmu.ac.uk

Citation: Al-Gazi MK, Carroll M (2015) Sperm-Specific MicroRNAs - Their Role and Function. J Genet Genomic Sci 1: 003.

Received: August 28, 2015; Accepted: October 07, 2015; Published: October 22, 2015

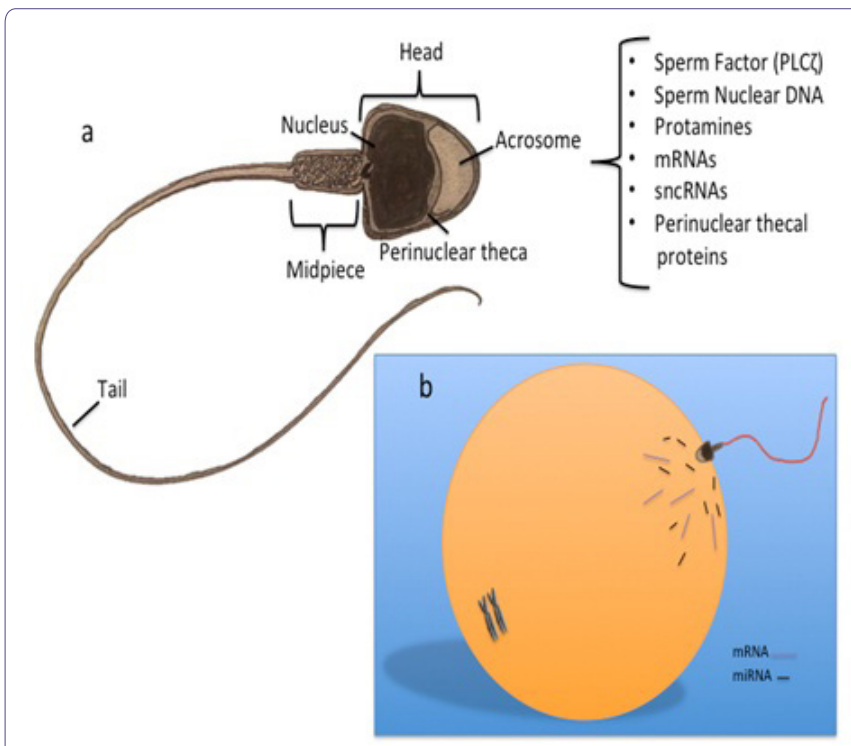

Figure 1: Sperm structure and constituents transferred to the oocyte at fertilization.

a) The fertilising sperm introduces more than the paternal genome, the

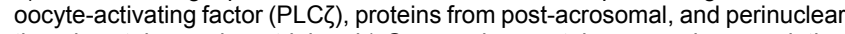
thecal proteins and centrioles; b) Sperm also contain a complex population of RNA, including messenger RNA (mRNA) transcripts, small non-coding RNAs (sncRNA) and microRNAs (miRNA), which are also introduced in to the ooplasm at fertilization.

\section{microRNAs}

miRNAs are small non-coding single-strand RNA molecules with sizes ranging from 17-26 nucleotides. The first miRNA to be discovered was lin-4 in the nematode, Caenorhabditis elegans. Lin-4 was detected with conserved complementary site on lin-14 and subsequently affected the larvae developmental timing by targeting mRNA and inhibiting its function [1]. Since then, miRNAs have emerged to play key roles in many biological functions through binding to 3'UTR (Untranslated Region) of specific mRNAs leading to either mRNA degradation or protein translation inhibition [2]. These miRNAs are involved in regulating cell functions including gene expression during development, differentiation, cell proliferation and apoptosis $[3,4]$.

In addition to having an influence in normal biological and developmental processes, miRNAs have been found to be involved in many pathogenic processes including Cardiovascular Diseases (CVD), cancer, inflammatory or autoimmune diseases, metabolic disorders and neurodegenerative disorders [5].

\section{miRNA biogenesis}

miRNAs are encoded in the genome and transcribed by RNA polymerase II to form long primary miRNA transcripts (pri-miRNAs) that contain a cap structure at the 5' end and are poly-adenylated at the 3' end.The primary miRNAs are subsequently processed in the nucleus by Drosha to form shorter stem loop structures called pre curser-miRNAs or pre-miRNA (figure 2). These pre-miRNAs are then 
transported to the cytoplasm by an Exportin-5 and processed by the RNase III enzyme called Dicer forming a short double-stranded miRNA duplex. This mature miRNA assembles into a protein-RNA complex called RISC (RNA Induced Silencing Complex) containing members of the Argonaut family. This multicomponent complex targets mRNA transcripts inhibiting gene expression by inhibiting ribosomal attachment, or through degradation of the mRNA transcript [6-8].

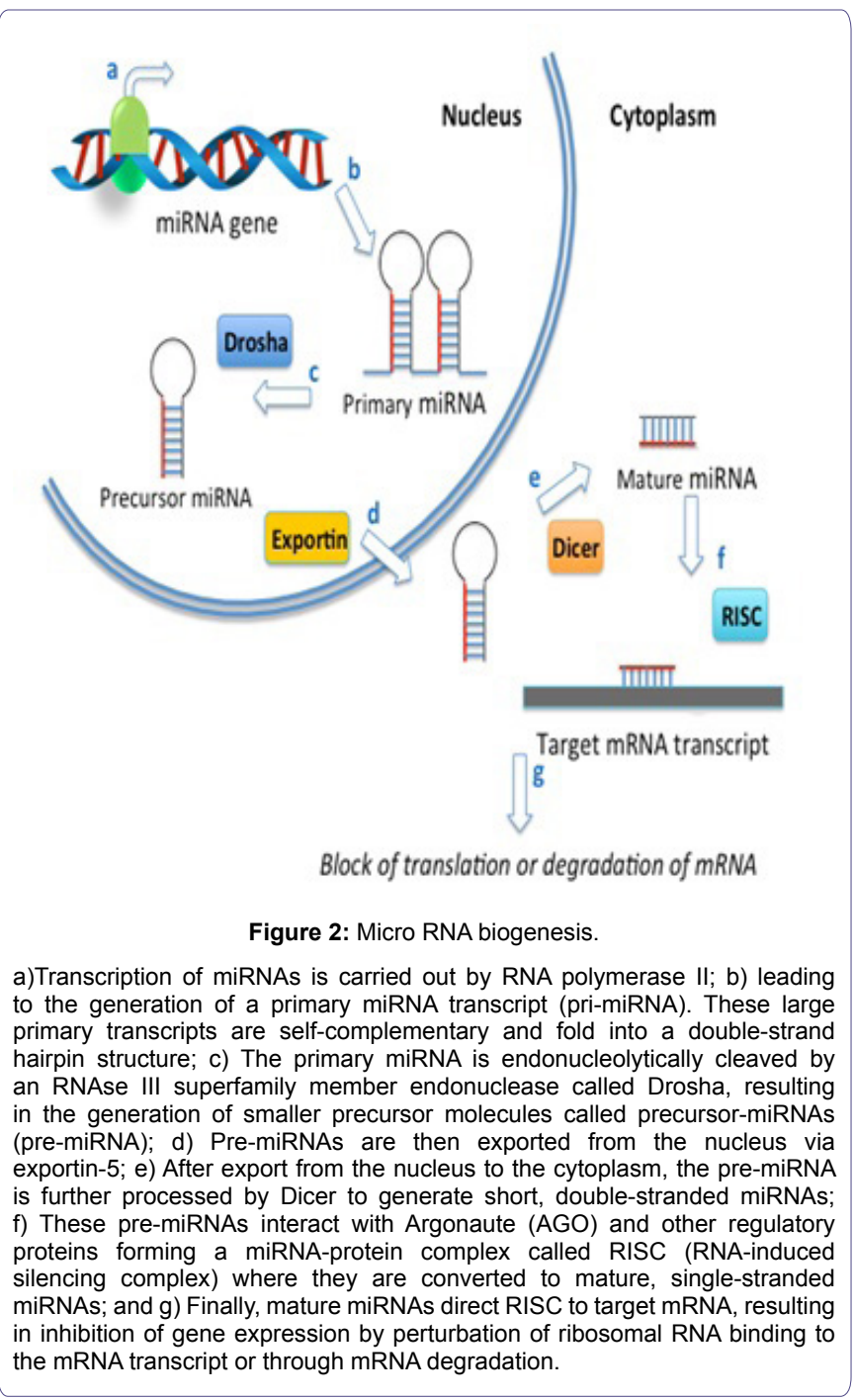

\section{Sperm-Specific miRNAs and Male Infertility}

The prevalence of infertility is estimated to be $15 \%$ globally, with males accounting to approximately $50 \%$ of cases. The causes of Male Factor Infertility (MFI) include infections, testicular injury, endocrinopathies and genetic disorders. However, a significant proportion of MFI is considered idiopathic, which is accompanied with low sperm numbers (oligozoospermia) and/or poor sperm motility (asthenozoospermia) [9].

Sperm contains a complex RNA population including messenger RNA (mRNA), transfer RNA (tRNA) and various non-coding RNAs (ncRNAs) such as the miRNAs [10]. The advancements in sequencing technology have enabled researchers to explore, in depth, the microRNA profile of many cell types [11], including sperm, where a plethora of novel sperm-specific miRNAs have been described
$[12,13]$. Increased miRNA levels at the pachytene spermatocyte stage and the post-meiotic stage during spermatid development together with specific miRNAs expression in mouse testis suggesting that miRNAs have a role in regulating spermatogenesis [14-17] Nixon et al has recently provided novel data for the post-testicular modification of sperm miRNA profile under normal physiological conditions. These modifications include both loss and acquisition of 113 and 115 miRNAs, respectively, between the proximal and distal epididymal segments. It was noted that the majority of these changes occur late in sperm maturation and include the uptake of novel miRNA species in addition to a significant increase in many miRNAs natively expressed in immature sperm [18].

Many reports have implicated dysregulation of sperm-specific miRNAs as likely to play an essential role in male fertility. In one study 154 differentially down regulated and 19 up-regulated miRNAs were found between a non-obstructive azoospermic group and a control group [19]. Additionally, it was noted that miRNA levels in seminal plasma revealed 19 miRNAs with altered expression in the seminal plasma and suggested 7 miRNAs that could be used as biomarkers of male infertility [20].

One study described 50 miRNAs up-regulated and 27 miRNAs down-regulated in as the no azoospermic males. In oligo-asthenozoospermic males (reduced sperm number and motility) 42 miRNAs were up-regulated and 44 miRNAs down-regulated when compared with normozoospermic males [21]. They showed miRNAs that exhibited the highest fold changes were miR-34b, miR-122, and miR-1973 in samples from asthenozoospermic men and miR-34b, miR-34b, miR-15b, miR-34c-5p, miR-122, miR-449a, miR-1973, miR-16, and miR-19a in samples from oligo-asthenozoospermic men. These data revealed a comprehensive number of miRNAs that were differentially expressed in asthenozoospermic and oligo-asthenozoospermic males compared with normozoospermic males, and provide support for the analysis of miRNA profiles as a future diagnosing tool for male infertility.

Another study compared the miRNA expression profile of sperm from three different infertile populations and one fertile group of men. They demonstrated that sperm from patients with seminal alterations exhibit a differential miRNA profile. Evaluating 736 miRNAs in these sperm, they found that hsa-miR-34b-3p correlated with age, the hsa-miR-629-3p with sperm motility, and the hsa-miR-335-5p, hsa-miR-885-5p, and has-miR-152-3p with sperm concentration [22].

Recently, it was demonstrated that dysregulation of miRNAs (miR-29 and miR-424) can induce DNA double-strand breaks during spermatogenesis - further highlighting the increasing role miRNAs have on sperm integrity and function [23].

In addition to influencing spermatogenesis and sperm function sperm-specific RNAs are introduced into the oocyte upon fertilization where it can contribute to gene regulation and embryo development [24].

Amanai et al, demonstrated that mature spermatozoa in mice contain a broad profile of miRNAs, and that potential mRNA targets of these miRNAs are expressed in metaphase II oocytes [25]. Early studies with human sperm revealed a unique population of sperm-specific miRNA, which are postulated to regulate sperm function [26] and may play an important role early zygotic and embryonic development [27]. 


\section{Sperm miRNA and Transgenerational Inheritance}

Epigenetic Transgenerational Inheritance (ETI) is defined as germ line transmission of epigenetic information between generations, via gametes, in the absence of alterations in the DNA sequence, and epigenetic modifications, such as chromatin / histone modification, DNA methylation and acetylation can contribute to alter gene expression in heritable manner without affecting the underlying genomic sequences [28,29]. There is increasing development highlighting the role of ncRNAs in ETI and in particular the role of sperm-borne ncRNAs such as miRNAs [30].

miRNAs are abundant in the mature sperm in mammals, and are introduced to the oocyte at fertilization, where they may transfer transgenerational inheritance [31]. There have been several interesting studies exploring the link between ETI (through transmission of sperm miRNAs) and environmental exposures. For example, paternal exposure to tobacco smoke and its components can alter the level of microRNAs in sperm [32]. Another study revealed that the predominant pathways mediated by miRNAs differentially expressed in the sperm of smokers were involved in cell proliferation, differentiation and death, pathways important during spermatogenesis and early embryo development. Thus, indicating potential epigenetic role for miRNAs in the multigenerational toxicity of cigarette smoke [33]. Diet can also alter miRNA expression and have transgenerational consequences as demonstrated in a study conducted by Fullston et al (2013). This study revealed that diet-induced paternal obesity modulates sperm miRNA content, which initiate the transmission of obesity and impaired metabolic health to future generations [34]. Furthermore, a recent study showed ETI through sperm-borne miRNA induced metabolic disorders in female offspring and with some intervention (diet and exercise) improved their metabolic health and prevents the metabolic syndrome in their female offspring [35].

Gapp et al, demonstrated that RNA-dependent processes contribute to the transmission of acquired traits in mammals. They carried out elaborate experiments and found that traumatic stress in early life altered mouse sperm miRNA expression, and behavioural and metabolic responses in the progeny. Injection of sperm miRNAs from traumatized males into fertilized wild-type oocytes reproduced the behavioural and metabolic alterations in the resulting offspring [36].

\section{miRNAs as a Biomarker for Male Infertility}

Profiling miRNAs is important in understanding how they regulate different biological pathways and contribute to disease, including infertility. Reliable and reproducible research on miRNA function depends on sample isolation, amplification and analysis specifically designed for miRNAs. There are several companies supplying kits explicitly for miRNA isolation and identification. These kits enable the investigator to isolate high quality miRNA for downstream applications. Once isolated the miRNA is reverse transcribed, converting all of the miRNA in to cDNA. These cDNA libraries can be used as templates for quantitative (Real Time) Polymerase Chain Reaction (qPCR)to profile the miRNA [37].

miRNAs can be isolated and detected in bodily fluids such as plasma, saliva, semen and vaginal fluid [38]. Therefore, utilizing this technology to explore the link between miRNA expression and male infertility can have great potential as a non-invasive biomarker for male infertility [39]. miRNAs and other noncoding
RNAs can be transported in the semen via seminal exosomes where they may be transferred to other cells with potential regulatory functions [40]. There are a growing numbers of studies reporting miRNA profiling in sperm form fertile and infertile males. One study described five miRNAs (has-miR-34b, has-miR-34b, has-miR-34c$5 \mathrm{p}$, has-miR-429, and has-miR-122) using qPCR analysis in sub fertile and non obstructive azoospermic patients, and control subjects. They reported that has-miR-429 was significantly increased and the four other miRNAs were decreased in both tested groups compared with normal control subjects. This study concluded that these five miRNAs have potential as novel non-invasive biomarkers to diagnose patients with sub fertility [41]. In another study the expression level of 736 miRNAs was evaluated. The investigators reported that up-regulated miRNAs presented an enriched localization in introns, affecting relevant genes for spermatogenesis and the predicted targets of the miRNAs contained critical genes associated to infertility [22]. miRNAs can also be a predictor for sperm DNA damage, as demonstrated by Zhao et al (2015), where they showed that miR-424/322 is involved in sperm DNA damage, and that the dysregulation of this miRNA can induce DNA double-strand breaks during spermatogenesis [23].

Human sperm contain a unique family of miRNA, which is responsible for normal spermatogenesis and sperm function and their expression profile will serve as a sensitive, selective and non-invasive diagnostic tests for male infertility.

\section{Conclusion}

The field of andrology has greatly expanded beyond the diagnostic field, merging with genetics, molecular biology and evolutionary biology. The sperm cell bestows to the oocyte much more than the paternal genome, centrioles and the activating factor - it provides the oocyte with a molecular signature that can influence not only early embryogenesis, but also determine the health of the progeny. miRNAs have shown to be of immense importance in many cellular processes and we are only at the beginning of our understanding of their role in both health and disease.

\section{References}

1. Lee RC, Feinbaum RL, Ambros V (1993) The C. elegans heterochronic gene lin-4 encodes small RNAs with antisense complementarity to lin-14. Cell 75: 843-854.

2. Guo H, Ingolia NT, Weissman JS, Bartel DP (2010) Mammalian microRNAs predominantly act to decrease target mRNA levels. Nature 466: 835-840.

3. Bartel DP, Chen CZ (2004) Micromanagers of gene expression: the potentially widespread influence of metazoan miRNAs. Nat Rev Genet 5: 396-400.

4. Yerramilli S, Shi P, Kreutz M, Qin J, Winter S, et al. (2013) Integrated expression profiling of multiple RNA species by real-time PCR. Methods 59: 7-10.

5. Ardekani AM, Naeini MM (2010) The Role of MicroRNAs in Human Diseases. Avicenna J Med Biotechnol 2: 161-179.

6. Winter J, Jung S, Keller S, Gregory RI, Diederichs S (2009) Many roads to maturity: microRNA biogenesis pathways and their regulation. Nat Cell Biol 11: 228-234.

7. Almeida MI, Reis RM, Calin GA (2011) MicroRNA history: discovery, recent applications, and next frontiers. Mutat Res 717: 1-8.

8. Ha M, Kim VN (2014) Regulation of microRNA biogenesis. Nat Rev Mol Cell Biol 15: 509-524.

9. Department of Reproductive Health and Research (2010) WHO Laboratory manual for the examination and processing of human semen (5thedn). Department of Reproductive Health and Research,World Health Organization, Geneva, Switzerland. 
10. Hamatani T (2012) Human spermatozoal RNAs. Fertil Steril 97: 275-281.

11. Barad O, Meiri E, Avniel A, Aharonov R, Barzilai A, et al. (2004) MicroRNA expression detected by oligonucleotide microarrays: system establishment and expression profiling in human tissues. Genome Res 14: 2486-2494.

12. Ostermeier GC, Dix DJ, Miller D, Khatri P, Krawetz SA (2002) Spermatozoal RNA profiles of normal fertile men. Lancet 360: 772-777.

13. Du Y, Wang X, Wang B, Chen W, He R, et al. (2014) Deep sequencing analysis of microRNAs in bovine sperm. Mol Reprod Dev 81: 1042-1052.

14. Yan N, Lu Y, Sun H, Tao D, Zhang S, et al. (2007) A microarray for microRNA profiling in mouse testis tissues. Reproduction 134: 73-79.

15. Ro S, Park C, Sanders KM, McCarrey JR, Yan W (2007) Cloning and expression profiling of testis-expressed microRNAs. Dev Biol 311: 592-602.

16. Miller D, Briggs D, Snowden H, Hamlington J, Rollinson S, et al. (1999) A complex population of RNAs exists in human ejaculate spermatozoa: implications for understanding molecular aspects of spermiogenesis. Gene 237 385-392.

17. Bouhallier F, Allioli N, Lavial F, Chalmel F, Perrard MH, et al. (2010) Role of miR-34c microRNA in the late steps of spermatogenesis. RNA 16: 720-731.

18. Nixon B, Stanger SJ, Mihalas BP, Reilly JN, Anderson AL, et al. (2015) The MicroRNA Signature of Mouse Spermatozoa is Substantially Modified During Epididymal Maturation. Biol Reprod 93: 91.

19. Lian J, Zhang X, Tian H, Liang N, Wang Y, et al. (2009) Altered microRNA expression in patients with non-obstructive azoospermia. Reprod Biol Endocrinol 7: 13.

20. Wang C, Yang C, Chen X, Yao B, Yang C, et al. (2011) Altered profile of seminal plasma microRNAs in the molecular diagnosis of male infertility. Clin Chem 57: 1722-1731.

21. Abu-Halima M, Hammadeh M, Schmitt J, Leidinger P, Keller A, et al. (2013) Altered microRNA expression profiles of human spermatozoa in patients with different spermatogenic impairments. Fertil Steril 99: 1249-1255.

22. Salas-Huetos A, Blanco J, Vidal F, Godo A, Grossmann M, et al. (2015) Spermatozoa from patients with seminal alterations exhibit a differential micro-ribonucleic acid profile. Fertil Steril 104: 591-601.

23. Zhao K, Chen Y, Yang R, Bai Y, Li C, et al. (2015) miR-424/322 is downregulated in the semen of patients with severe DNA damage and may regulate sperm DNA damage. Reprod Fertil Dev.

24. Sendler E, Johnson GD, Mao S, Goodrich RJ, Diamond MP, et al. (2013) Stability, delivery and functions of human sperm RNAs at fertilization. Nucleic Acids Res 41: 4104-4117.

25. Amanai M, Brahmajosyula M, Perry AC (2006) A restricted role for spermborne microRNAs in mammalian fertilization. Biol Reprod 75: 877-884.

26. Ostermeier GC, Miller D, Huntriss JD, Diamond MP, Krawetz SA (2004) Reproductive biology: delivering spermatozoan RNA to the oocyte. Nature 429 : 154
27. Boerke A, Dieleman SJ, Gadella BM (2007) A possible role for sperm RNA in early embryo development. Theriogenology 1: 147-155.

28. Reik W, Dean W, Walter J (2001) Epigenetic reprogramming in mammalian development. Science 293: 1089-1093.

29. Trerotola M, Relli V, Simeone P, Alberti S (2015) Epigenetic inheritance and the missing heritability. Hum Genomics 9: 17.

30. Yan W (2014) Potential roles of noncoding RNAs in environmental epigenetic transgenerational inheritance. Mol Cell Endocrinol 398: 24-30.

31. Kawano M, Kawaji H, Grandjean V, Kiani J, Rassoulzadegan M (2012) Novel small noncoding RNAs in mouse spermatozoa, zygotes and early embryos. PLoS One 7: 44542.

32. Metzler-Guillemain C, Victorero G, Lepoivre C, Bergon A, Yammine M, et al (2015) Sperm mRNAs and microRNAs as candidate markers for the impact of toxicants on human spermatogenesis: an application to tobacco smoking. Syst Biol Reprod Med 61: 139-149.

33. Marczylo EL, Amoako AA, Konje JC, Gant TW, Marczylo TH (2012) Smoking induces differential miRNA expression in human spermatozoa: a potentia transgenerational epigenetic concern? Epigenetics 7: 432-439.

34. Fullston T, Ohlsson Teague EM, Palmer NO, DeBlasio MJ, Mitchell M, et al. (2013) Paternal obesity initiates metabolic disturbances in two generations of mice with incomplete penetrance to the F2 generation and alters the transcriptional profile of testis and sperm microRNA content. FASEB J 27: 4226-4243.

35. McPherson NO, Owens JA, Fullston T, Lane M (2015) Preconception diet or exercise intervention in obese fathers normalizes sperm microRNA profile and metabolic syndrome in female offspring. Am J Physiol Endocrinol Metab 308: 805-821.

36. Gapp K, Jawaid A, Sarkies P, Bohacek J, Pelczar P, et al. (2014) Implication of sperm RNAs in transgenerational inheritance of the effects of early trauma in mice. Nat Neurosci 17: 667-669.

37. Park JL, Park SM, Kwon OH, Lee HC, Kim JY, et al. (2014) Microarray screening and qRT-PCR evaluation of microRNA markers for forensic body fluid identification. Electrophoresis 35: 3062-3068

38. Lässer C (2013) Identification and analysis of circulating exosomal microRNA in human body fluids. Methods Mol Biol 1024: 109-128.

39. Etheridge A, Lee I, Hood L, Galas D, Wang K (2011) Extracellular microRNA: a new source of biomarkers. Mutat Res 717: 85-90.

40. Vojtech L, Woo S, Hughes S, Levy C, Ballweber L, et al. (2014) Exosomes in human semen carry a distinctive repertoire of small non-coding RNAs with potential regulatory functions. Nucleic Acids Res 42: 7290-7304.

41. Abu-Halima M, Hammadeh M, Backes C, Fischer U, Leidinger $P$, et al. (2014) Panel of five microRNAs as potential biomarkers for the diagnosis and assessment of male infertility. Fertil Steril 102: 989-997. 